\title{
Adsorción de Fenol y 3-Cloro Fenol sobre Carbones Activados mediante Calorimetría de Inmersión
}

\author{
Juan C. Moreno-Piraján ${ }^{(1) *}$, Luisa F. Navarrete ${ }^{(2)}$, Liliana Giraldo ${ }^{(2)}$ y Vanessa García(1) \\ (1) Grupo de Investigación en sólidos porosos y calorimetría, Departamento de Química, \\ Fac. de Ciencias, Universidad de Los Andes, Carrera 1 No. 18 A 10, Bogotá-Colombia. \\ (e-mail: jumoreno@uniandes.edu.co) \\ (2) Departamento de Química, Facultad de Ciencias, Universidad Nacional de Colombia, \\ Ciudad Universitaria, Carrera 30 No. 45 03, edificio 451, Bogotá-Colombia.
}

* autor a quien debe ser dirigida la correspondencia

\section{Resumen}

Se ha determinado la entalpía de inmersión de cinco muestras de carbón activado, obtenidas a partir de diferentes materiales lignocelulósicos, con diferente grado de activación en disoluciones acuosas de fenol y 3-cloro fenol con el fin de caracterizar la interacción sólido-disolución acuosa, obteniéndose valores entre 6.80 y $13.9 \mathrm{Jg}-1$. Se determinó también, las entalpías de inmersión de estos carbones activados en disoluciones de $\mathrm{NaOH}$ y $\mathrm{HCl} 0.1 \mathrm{M}$, obteniéndose los más altos valores de la entalpía de inmersión en $\mathrm{HCl}$, entre 32.6 y $68.3 \mathrm{Jg}^{-1}$. Los valores de las entalpías de inmersión disminuyen con el aumento en el contenido de grupos ácidos superficiales de los carbones activados, y resulta ser el factor determinante para la adsorción del fenol y el 3-cloro fenol en los sólidos estudiados.

Palabras clave: adsorción de fenol, acidez y basicidad, calorimetría de inmersión, entalpía de inmersión

\section{Adsorption of Phenol and 3-Chlorophenol n Activated Carbons using Immersion Calorimetry}

\begin{abstract}
Immersion enthalpies of five samples of activated carbon obtained from diverse lignocellulosic materials are determined, each with different activation degree in aqueous solutions of phenol and 3chlorophenol for characterising the solid-aqueous dissolution interaction. The values obtained were between 6.80 y $13.9 \mathrm{Jg}-1$. Also, immersion enthalpies of these activated carbons in $\mathrm{NaOH}$ and $\mathrm{HCl}$ $0.1 \mathrm{M}$ solutions were determined, obtaining higher values for the immersion enthalpy in $\mathrm{HCl}$ that ranged between 32.6 and $68.3 \mathrm{Jg}^{-1}$. Immersion enthalpies values decrease with the increase of superficial acid group content of the activated carbons, which constitutes the determinant factor for the phenol and 3-chlorophenol adsorption in the solids studied.
\end{abstract}

Keywords: phenol adsorption, acidity and basicity, immersion calorimetry, immersion enthalpy 


\section{INTRODUCCION}

Los procesos de adsorción en fase líquida se utilizan con buena eficiencia en la purificación de aguas residuales de industrias que pueden contener colorantes, fragancias y en general contaminantes orgánicos o inorgánicos (Tseng et al., 2003). Los procesos de urbanización e industrialización que se han realizado en forma general en todos los países y que han aportado grandes beneficios a la población, han generado también, muchas veces por falta de planeación, serios problemas ambientales como son la alteración de los ecosistemas, el manejo inadecuado de recursos naturales, la contaminación ambiental y daños a la salud de la población humana. Las descargas de los efluentes que contienen compuestos tóxicos, aun en bajas concentraciones, han ocasionado la contaminación de los suelos, los mantos freáticos, los acuíferos profundos, las aguas continentales y costeras. Las aguas residuales que contienen metales y compuestos orgánicos tóxicos representan una fuente importante de contaminación (McGuire et. al., 1983; De Zuane, 1997; Otero et. al., 2005; Rostaei et.al., 2004; Nemerow, 1991).

El carbón activado es un adsorbente que presenta un elevado y variado grado de porosidad, una considerable superficie interna y un cierto contenido de grupos químicos superficiales; estas características son las responsables de sus propiedades adsorbentes, utilizadas en aplicaciones tanto en fase gaseosa como en fase líquida. El carbón activado es un adsorbente muy versátil, porque el tamaño y distribución de sus poros en la estructura carbonosa pueden ser controlados para satisfacer las necesidades de purificación en fase gaseosa y líquida (Moreno-Castilla, 2004; Nevskaia et. al., 2004; Mozia et. al., 2005).

Una de las aplicaciones de los carbones activados es la de eliminar compuestos orgánicos de aguas, y para esta aplicación particular uno de los aspectos que tiene mayor influencia en la adsorción es la química de la superficie (Cartula et al., 1998; McGuire et al., 1983), la que depende del contenido de heteroátomos, principalmente grupos con oxígeno; que determinan en los carbones activados la carga de la superficie, la hidrofobicidad y la densidad electrónica de las capas grafénicas. Los grupos químicos en la superficie de los carbones activados hacen que las partículas en solución presenten una carga eléctrica que influye en los procesos de adsorción; y que se relaciona con el $\mathrm{pH}$ en el punto de carga cero, $\mathrm{pH}_{\text {PzC }}$, que cuantifica la carga superficial total. Cuando el $\mathrm{pH}$ de la solución es menor que el $\mathrm{pH}_{\mathrm{PzC}}$ del sólido la carga total será positiva, mientras que si el pH de la solución es mayor que el $\mathrm{pH}_{\mathrm{PzC}}$ la superficie estará cargada negativamente (Radovic et al., 2000). La interacción entre carbón activado y fenoles presentes en soluciones acuosas se puede determinar por medio de la entalpía de inmersión del sólido en soluciones que contengan sustancias activas con la superficie y que permiten relacionar las propiedades fisicoquímicas del carbón activado con la capacidad de adsorción de los compuestos que están en solución (Giraldo et. al, 2003; Lopez et al., 1999).

En este trabajo se estudian cinco muestras de carbón activado, obtenidos a partir de materiales lignocelulósicos mediante activación física, con porcentajes de activación alrededor de $20 \%$, a los que se les determina el punto de carga cero, el contenido total de grupos ácidos y básicos totales, la adsorción y entalpía de inmersión en soluciones acuosas de fenol y 3-cloro fenol. En un trabajo anterior se determinaron la cantidad adsorbida y la entalpía de inmersión para de 4-nitro fenol con los de carbones activados utilizados en este trabajo y se compara con los resultados de fenol y 3-cloro fenol (Navarrete et. al 2006).

\section{METODOLOGIA}

Los carbones activados se han preparado mediante carbonización en atmósfera de nitrógeno y posterior activación con $\mathrm{CO}_{2}$ a $950{ }^{\circ} \mathrm{C}$ por un período de 3 horas, en estas condiciones se obtienen materiales con porcentajes de activación del orden de 22-33 \%, como es el caso de las muestras que se utilizan en este trabajo, y que corresponde a un desarrollo de área superficial moderado (Rodríguez, 1997). Al preparar los carbones activados con las mismas condiciones experimentales, las características superficiales finales de cada uno de éstos se deben a la naturaleza del material precursor (Briceño 2002, Díaz et.al. 2002, Amaya et.al., 2004). En la Tabla 1 se presentan la procedencia, denominación, porcentaje de activación y área superficial de los carbones activados que se emplean en este trabajo. 
Tabla 1: Características de los carbones activados

\begin{tabular}{|c|c|c|c|}
\hline Precursor & Denominación & Porcentaje Activación & Area superficial $\left(\mathrm{m}^{2} \mathrm{~g}^{-1}\right)$ \\
\hline $\begin{array}{c}\text { Cuesco de palma } \\
\text { africana }\end{array}$ & CACu & 18 & 274 \\
\hline Cáscara de coco & CACo & 21 & 292 \\
\hline Carbón mineral & CAM & 28 & 435 \\
\hline $\begin{array}{c}\text { Cascarilla de café } \\
\text { Bagazo de caña de } \\
\text { azúcar }\end{array}$ & CACf & 24 & 338 \\
\hline
\end{tabular}

Determinación de los Sitios Ácidos y Básicos.

Se determinan la acidez y la basicidad total de los carbones activados por el método de Boehm (Boehm H.P., 1966). Se pesa aproximadamente $1.000 \mathrm{~g}$ de cada sólido para cada determinación: una cantidad de carbón activado se coloca en un frasco de vidrio con tapa esmerilada con $50.0 \mathrm{~mL}$ de una disolución de hidróxido de sodio, $\mathrm{NaOH}, 0.1 \mathrm{M}$ y otra cantidad se coloca en un recipiente con $50.0 \mathrm{~mL}$ de una disolución de ácido clorhídrico, $\mathrm{HCl}, 0.1 \mathrm{M}$. Las disoluciones se mantienen a $25.0^{\circ} \mathrm{C}$, durante 5 días. Se toman muestras alícuotas de $10.0 \mathrm{~mL}$ de cada una de las disoluciones y se titula correspondientemente: la disolución de $\mathrm{NaOH}$ con $\mathrm{HCl}$ y la disolución de $\mathrm{HCl}$ con $\mathrm{NaOH}$ de las concentraciones mencionadas, con un pH meter CG 840B Schott.

Determinación del Punto de Carga Cero, PCZ. Método de Titulación de Masas.

Se pesan cantidades de los carbones activados en un rango de 0.010 a $0.600 \mathrm{~g}$, cada una de las cuales se coloca en un frasco de vidrio de $50.0 \mathrm{~mL}$ y se le agrega $10.0 \mathrm{~mL}$ de disolución de cloruro de sodio, $\mathrm{NaCl}, 0.1 \mathrm{M}$. Los frascos se tapan y se dejan en agitación a temperatura constante de 25.0 ${ }^{\circ} \mathrm{C}$ durante 48 horas, posteriormente se mide el $\mathrm{pH}$ de cada una de las disoluciones (Giraldo et.al., 2004).

Determinación de la cantidad de fenol y 3-cloro fenol adsorbidos.

Para determinar la cantidad que adsorbe cada carbón activado de fenol y de 3-cloro fenol se emplea el adsorbedor que se muestra en la figura 1 , donde se colocan $0.500 \mathrm{~g}$ de cada carbón activado en frascos de vidrio y $250 \mathrm{~mL}$ de las respectivas disoluciones acuosas de fenol y 3-cloro fenol con una concentración de $100 \mathrm{mgL}^{-1}$.

Las muestras se agitan mecánicamente y se mantienen a una temperatura de $25.0{ }^{\circ} \mathrm{C}$, por un periodo de aproximadamente 72 horas. La concentración de equilibrio de los compuestos fenólicos en las disoluciones después de la adsorción se determina, previa calibración, con un equipo espectrofotométrico Uv-Vis Milton Roy Co. Spectronic Genesys SN.

Determinación de la entalpía de inmersión de los carbones activados en las disoluciones fenólicas.

En este trabajo se realizan determinaciones de la entalpía de inmersión de los carbones activados en varias disoluciones que son: $\mathrm{NaOH} 0.1 \mathrm{M}, \mathrm{HCl} 0.1 \mathrm{M}$ y las disoluciones acuosas de fenol y 3-cloro fenol de $100 \mathrm{mgL}^{-1}$. A continuación se hace una descripción general de la forma en que se llevan a cabo estas determinaciones. Para determinar las entalpías de inmersión se usa un microcalorímetro de conducción de calor, con una celda calorimétrica en acero inoxidable (Giraldo et al., 2002). 


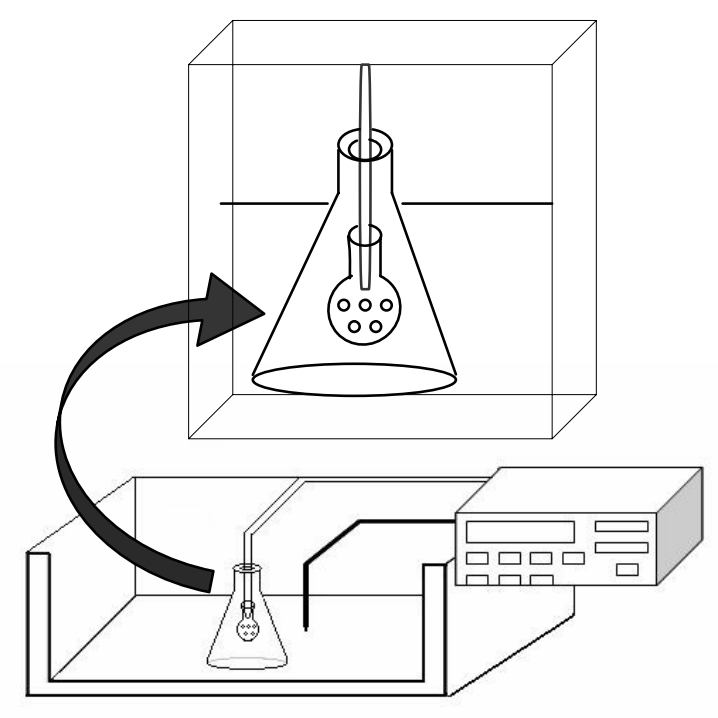

Fig. 1: Adsorbedor empleado para obtener las isotermas desde las soluciones fenólicas

Se colocan en la celda $20 \mathrm{~mL}$ de la disolución a utilizar, que se han mantenido en un termostato a $25.0^{\circ} \mathrm{C}$; se pesa una muestra de carbón activado del orden de $0.500 \mathrm{~g}$ y se coloca dentro de la celda calorimétrica en una ampolleta de vidrio, se ensambla el microcalorímetro. Cuando el equipo alcanza una temperatura de alrededor $25^{\circ} \mathrm{C}$, se inicia el registro de potencial de salida por un periodo de aproximadamente 15 minutos tomando lecturas de potencial cada 15 segundos, se procede a realizar el rompimiento de la ampolleta de vidrio, se registra el efecto térmico generado y se continua con las lecturas de potencial por aproximadamente 30 minutos más, finalmente se calibra eléctricamente.

\section{RESULTADOS Y DISCUSIÓN}

El proceso de activación al que se han sometido los materiales de partida para obtener los carbones activados hace que éstos tengan valores de área superficial, que están entre 274 y $435 \mathrm{~m}^{2} \mathrm{~g}^{-1}$, de tal forma que la diferencia es de alrededor del $160 \mathrm{~m}^{2} \mathrm{~g}^{-1}$, y se observa en los resultados de la adsorción de los compuestos fenólicos que éstos no son los carbones activados que presentan la mínima y la máxima adsorción; en la Figura 2 se presenta la relación entre la cantidad adsorbida de cada compuesto fenólico en los carbones activados en función del área superficial de éstos, las gráficas muestran que para los tres compuestos la relación no es lineal y por tanto la mayor adsorción se obtiene para el carbón activado CACf que tiene como precursor cascarilla de café y que presenta un área superficial intermedia, de $338 \mathrm{~m}^{2} \mathrm{~g}^{-1}$, del conjunto de sólidos que se emplea.

El análisis se centra entonces en la química superficial de los carbones activados, que depende en buena parte, de su contenido de heteroátomos, y éstos determinan la carga de la superficie, su hidrofobicidad, acidez total y basicidad total, y tales factores intervienen en la capacidad de adsorción del carbón activado (Radovic et al., 2000). Por esta razón se han cuantificado el contenido de grupos de superficie ácidos y básicos totales, la entalpía de inmersión de los carbones activados en disoluciones de ácido y base y se ha determinado el $\mathrm{pH}$ en el punto de carga cero, para relacionarlos con la adsorción de fenol y 3-cloro fenol en disolución acuosa. En la Tabla 2 se presentan los resultados obtenidos para las entalpías de inmersión de los carbones activados en disoluciones de $\mathrm{NaOH}$ y $\mathrm{HCl} 0.1 \mathrm{M}$, en $\mathrm{Jg}^{-1}$ y el $\mathrm{pH}_{\text {PzC }}$.

Los resultados obtenidos para los contenidos de grupos ácidos totales de las cinco muestras de carbones activados presentan valores de acidez en un rango aproximado de 0.15 a $0.52 \mathrm{meqg}^{-1}$ que permite apreciar diferencias entre éstos, que se reflejan en los valores de la entalpía de inmersión en disolución $0.10 \mathrm{M}$ de $\mathrm{NaOH}$; la basicidad total varía entre 0.53 y $0.79 \mathrm{meqg}^{-1}$ que muestra el carácter 
básico de los carbones activados obtenidos térmicamente en las condiciones descritas, sin embargo las diferencias entre la acidez y la basicidad no son grandes, en ningún caso son valores mayores a la unidad, y es un comportamiento comparable con los obtenidos en otros trabajos realizados por nuestro grupo con anterioridad y también por otros autores (Diaz et al., 2003; Toles et al., 1999).

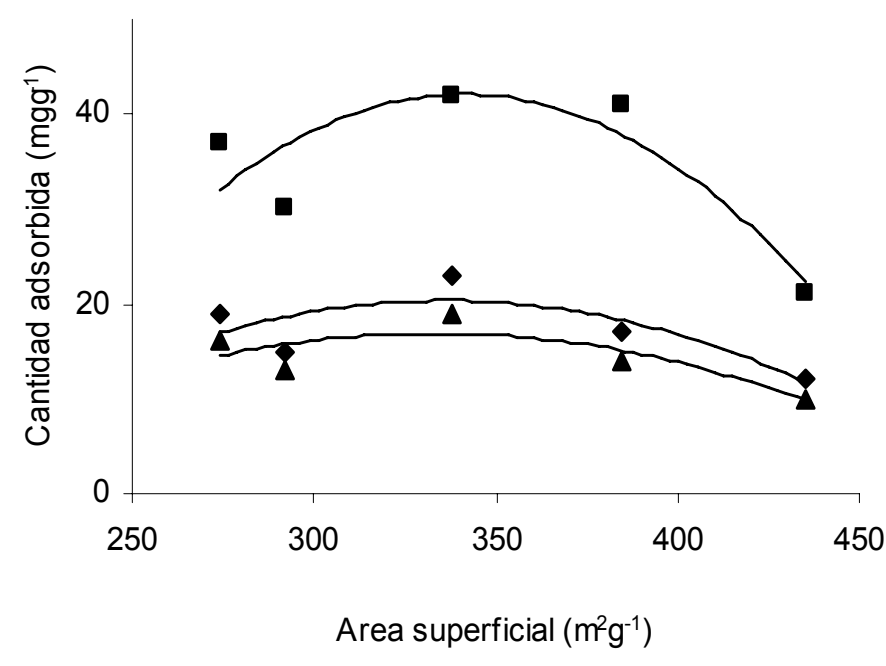

Fig. 2: Cantidad adsorbida de los compuestos fenólicos en función del área superficial de los carbones activados. fenol, - 4-nitro fenol, $\boldsymbol{\Delta}$ 3cloro fenol

Tabla 2: Características químicas superficiales de los carbones activados.

\begin{tabular}{|c|c|c|c|}
\hline Carbón activado & $\begin{array}{c}-\Delta \text { Hinm NaOH } \\
0.1 \mathrm{M}\left(\mathrm{Jg}^{-1}\right)\end{array}$ & $\begin{array}{c}-\Delta \mathrm{Hinm} \mathrm{HCl} \\
0.1 \mathrm{M}\left(\mathrm{Jg}^{-1}\right)\end{array}$ & $\mathrm{pH}_{\mathrm{PZC}}$ \\
\hline CACu & 18.6 & 32.6 & 8.9 \\
\hline CACo & 24.2 & 68.3 & 7.8 \\
\hline CAM & 28.5 & 49.0 & 7.4 \\
\hline CACf & 15.6 & 57.2 & 9.7 \\
\hline $\mathrm{CAB}$ & 20.3 & 51.3 & 9.2 \\
\hline
\end{tabular}

Para todos los carbones activados, su inmersión tanto en disolución de $\mathrm{NaOH}$ como de $\mathrm{HCl}$ genera valores de las entalpías de inmersión negativos, lo que indica que tal proceso es exotérmico, y con valores mayores para la inmersión en la disolución de ácido clorhídrico, confirmando nuevamente mayor interacción con los sitios básicos de los carbones activados. Los resultados para las entalpías de inmersión de la Tabla 2 corresponden al promedio de diez determinaciones y las desviaciones estándar se encuentran entre $0.20 \mathrm{y} 1.0 \mathrm{Jg}^{-1}$. En lo referente al $\mathrm{pH}$ en el punto de carga cero, $\mathrm{pH}_{\mathrm{PZC}}$, para todos los carbones activados éste es básico lo que los clasifica como carbones activados tipo $\mathrm{H}$; de tal forma que si los sólidos se ponen en contacto con disoluciones cuyo pH sea menor que el $\mathrm{pH}_{\mathrm{PzC}}$ la carga de su superficie será en promedio positiva, que es lo que ocurre en este trabajo dado que las determinaciones de cantidad de adsorción y calorimetría de inmersión se hacen sin ajuste del pH. (Moreno-Castilla, 2004)

En la Tabla 3 se resumen los resultados obtenidos para las entalpías de inmersión, $\Delta$ Hinm, de los carbones activados en disoluciones acuosas de fenol, 3-cloro fenol y 4-nitro fenol, obtenido en un trabajo anterior (Navarrete et.al, 2006), con una concentración de $100 \mathrm{mgL}^{-1}$, en $\mathrm{Jg}^{-1}$. Los resultados son el promedio de tres determinaciones y los valores para las desviaciones estándar se encuentran entre 0.30 y $1.71 \mathrm{Jg}^{-1}$. 
Como la adsorción del compuesto fenólico en el carbón activado se produce debido a que se favorecen determinadas interacciones que generan un cambio en la entalpía del sistema, estas dos propiedades: cantidad adsorbida y entalpía de inmersión se afectan por el contenido de grupos ácidos y básicos totales de la superficie de los carbones activados; se conoce que al disminuir el contenido de grupos ácidos la cantidad adsorbida del compuesto fenólico aumenta, dado que se interfiere menos con los electrones $\pi$ de las capas grafénicas de la superficie del carbón activado y que a su vez interactúan con los electrones $\pi$ del anillo aromático de los solutos (Moreno-Castilla, 2004). En la Figura 3, se muestra las gráficas de la cantidad adsorbida de los tres compuestos en los carbones activados cuyos contenidos de grupos ácidos varían.

Tabla 3: Entalpías de inmersión de los carbones activados en soluciones acuosas de fenol y 3-cloro fenol. ${ }^{a}$ Resultados de un trabajo anterior (Navarrete et.al, 2006)

\begin{tabular}{|c|c|c|c|}
\hline Carbón activado & $\begin{array}{c}-\Delta \text { Hinm } \\
\text { Fenol }\left(\mathrm{Jg}^{-1}\right)\end{array}$ & $\begin{array}{c}-\Delta \text { Hinm } \\
\text { 3-cloro fenol }\left(\mathrm{Jg}^{-1}\right)\end{array}$ & $\begin{array}{c}-\Delta \text { Hinm } \\
\text { 4-nitro fenol }\end{array}$ \\
\hline CACu & 10.2 & 8.70 & 18.6 \\
\hline CACo & 9.50 & 7.90 & 12.7 \\
\hline CAM & 7.60 & 6.80 & 10.3 \\
\hline CACf & 13.9 & 11.4 & 20.5 \\
\hline CAB & 12.4 & 9.10 & 17.8 \\
\hline
\end{tabular}

Se observa que la cantidad adsorbida en los carbones activados es en orden decreciente 4-nitro fenol, fenol y 3-cloro fenol y que las cantidades máximas se obtienen con el carbón activado CACf y las cantidades menores para el carbón activado CAM, Los que a su vez presentan el menor y mayor contenido de grupos ácidos respectivamente; la tendencia lineal del comportamiento se ajusta mejor al fenol y 3-cloro fenol, con coeficientes de correlación de 0.9673 y 0.9465 , y se observa una mayor dispersión para el caso de la adsorción del 4-nitro fenol con un coeficiente de correlación de 0.7845 , en el que el punto para el carbón activado obtenido a partir de caña de azúcar, CAB, se aleja de la tendencia.

Cuando se realiza la representación del contenido de grupos básicos con la cantidad adsorbida de los compuestos fenólicos, no se presenta un tipo de correlación similar, lo que puede indicar que el carácter básico en los materiales carbonosos tiene una influencia distinta en la adsorción y que está representada no solamente por los grupos funcionales de tipo básico sino también por la interacción electrónica (Montes-Moran et.al, 2004).

La entalpía de inmersión es un indicativo de la interacción entre el sólido y el líquido de inmersión, que en este trabajo es la disolución acuosa del compuesto fenólico, el valor de esta propiedad corresponde a la cantidad total de calor que se genera en el proceso de adsorción del soluto y por tanto implica también la interacción del solvente con el sólido; en la Figura 4 se muestran las gráficas de la entalpía de inmersión de los carbones activados en función del contenido de grupos ácidos.

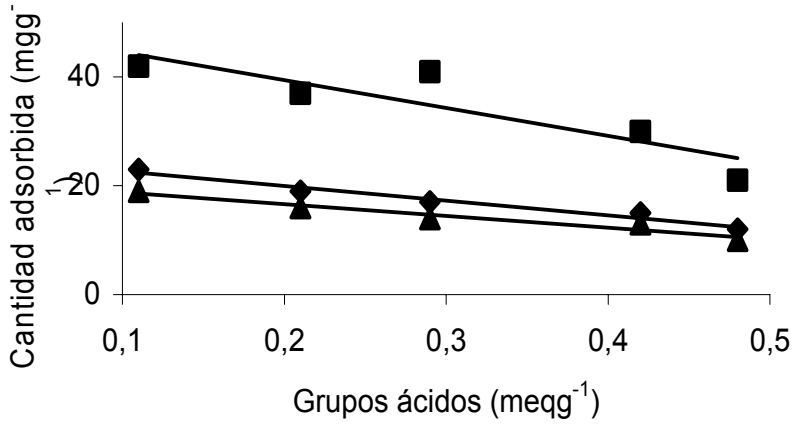

Fig. 3: Cantidad adsorbida de los compuestos fenólicos en carbones activados con diferente contenido de grupos ácidos. • fenol, - 4-nitro fenol, $\Delta$ 3-cloro fenol.

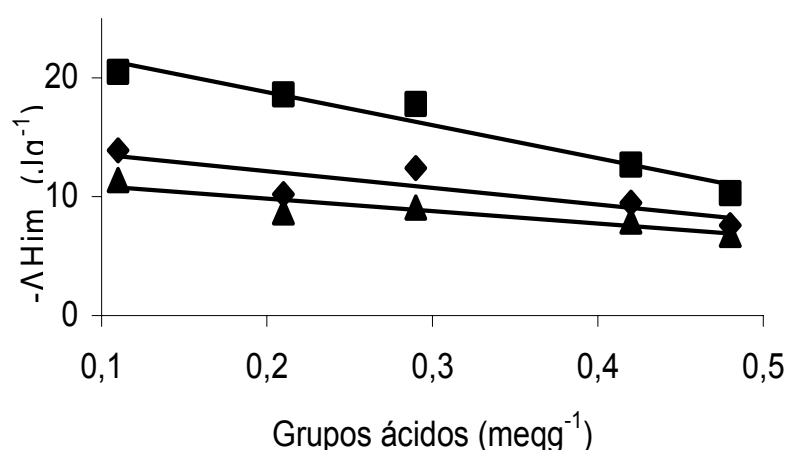

Fig. 4: Entalpía de inmersión de carbones activados en soluciones acuosas de compuestos. fenol, घ 4-nitro fenol, $\boldsymbol{\Delta}$ 3-cloro fenol. 
Como se observa en las gráficas se presenta, al igual que en la Figura 3, una relación lineal entre la entalpía de inmersión de los carbones activados en disoluciones de $100 \mathrm{mgL}^{-1}$ de cada uno de los compuestos fenólicos y el contenido de grupos ácidos de la superficie. Los valores de la entalpía de inmersión son negativos y mayores para el caso del 4-nitro fenol, en tanto que las inmersiones en disoluciones de 3-cloro fenol son las que generan los menores valores en la entalpía de inmersión; estos resultados muestran que las interacciones son mayores en el primer caso y menores para el 3cloro fenol, lo que se puede relacionar con la presencia de los sustituyentes en el anillo aromático dado que el fenol presenta un comportamiento Intermedio. Resultados similares, con respecto a la cantidad adsorbida de los compuestos fenólicos se encuentran en literatura (Moreno-Castilla et.al.,1995), sin embargo es válido anotar que cuando se cambia las condiciones del pH de la solución los resultados son diferentes porque la condición de carga de los componentes del sistema es distinta (Radovic et.al., 2000). Los resultados obtenidos para la entalpía de inmersión confirman el hecho que se presenta una menor interacción sólido-líquido cuando se aumenta el contenido de sitios ácidos superficiales y que este propiedad tiene una mayor influencia que el área superficial del carbón activado.

Otro parámetro de caracterización de la superficie del carbón activado, que está relacionado con el contenido de los grupos superficiales es el $\mathrm{pH}$ en el punto de carga cero, $\mathrm{pH}_{\mathrm{Pzc}}$, y que permite establecer la carga promedio de la superficie del sólido dependiendo de las condiciones de $\mathrm{pH}$; se muestra el la Figura 5 la relación de este parámetro y la entalpía de inmersión de los carbones activados en las disoluciones de los solutos. Se observa en que al aumentar el valor del pHPZC se aumenta la entalpía de inmersión para los tres compuestos estudiados, siguiendo una tendencia lineal, con coeficientes de correlación de $0.9637,0.9004$ y 0.8511 para el 4-nitro fenol, fenol y 3-cloro fenol respectivamente. El carbón activado con el menor valor de $\mathrm{pH}_{\mathrm{PzC}}$ es el CAM y el que tiene el mayor valor es el CACf. Se aprecia que para el carbón activado con menor $\mathrm{pH}_{\mathrm{PZC}}$ y con mayor contenido de grupos ácidos la entalpía de inmersión en las disoluciones de los tres compuestos fenólicos presenta una menor diferencia que en los otros carbones activados, es decir las interacciones se hacen menores y por tanto también disminuye la cantidad adsorbida de los compuestos; este resultado es interesante porque al tener valores de las entalpías de inmersión cercanos, $6.80,7.60$ y $10.3 \mathrm{Jg}^{-1}$ para el 3-cloro fenol, fenol y 4-nitro fenol respectivamente, se observa que las interacciones se hacen menores y que están afectadas por la presencia de grupos oxigenados.

De los carbones activados estudiados los que presentan los valores extremos en la adsorción del fenol y el 3-cloro fenol son el CAM y el CACf, el primero presenta la menor adsorción de los dos compuestos y el segundo la mayor; por tanto se determina para estos dos carbones activados, la entalpía de inmersión a diferentes concentraciones de la disolución de 3-cloro fenol, ya que para este compuesto se tienen los menores valores de adsorción en la serie de carbones activados de este trabajo y de esta forma se puede observar como afecta la cantidad de soluto la entalpía de inmersión. En la Figura 6, se muestran las gráficas de entalpía de inmersión de los carbones activados CAM y CACf en función de la concentración de disolucines acuosas de 3-cloro fenol.

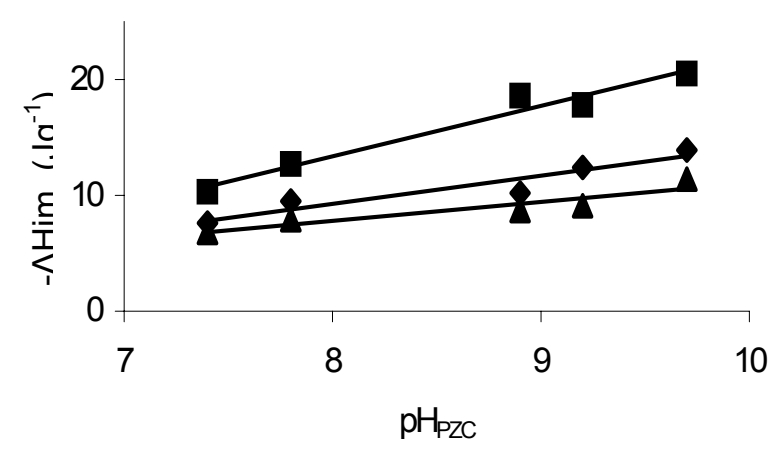

Fig. 5: Entalpía de inmersión de los carbones activados en disoluciones de compuestos fenólicos de $100 \mathrm{mgL}^{-1}$ en función del $\mathrm{pH}_{\mathrm{PZC}}$ - fenol, a 4-nitro fenol, $\Delta$ 3-cloro fenol

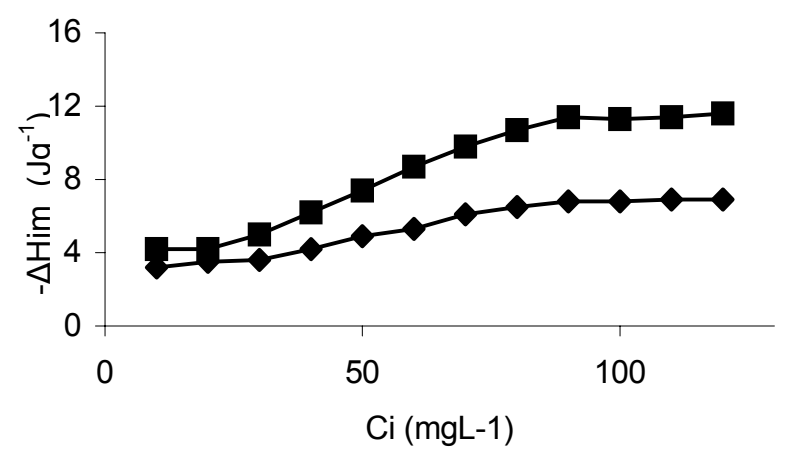

Fig. 6: Entalpía de inmersión de los carbones activados CAM y CACf en función de la concentración de 3-cloro fenol. Carbón activado CAM, - Carbón activado CACf 
Las determinaciones de la entalpía de inmersión de los dos carbones activados, se llevan a cabo en un rango de concentración de 10 a $120 \mathrm{mgL}^{-1}$, dado que para las últimas concentraciones los valores de la entalpía de inmersión se mantienen constantes; este resultado es interesante porque muestra que al aumentar a concentración se llega a un punto en que las interacciones entre el líquido y el sólido no se modifican y se mantienen en un valor constante, semejando la forma que se tiene en las isotermas de adsorción en fase acuosa. Resultados similares se han obtenido anteriormente para las entalpías de inmersión de un carbón activado comercial en soluciones acuosas de fenol (Giraldo et.al., 2003); estas determinaciones muestran que se llega a un equilibrio entre el sólido y la solución a concentraciones para las cuales la entalpía de inmersión se mantiene constante.

Si se toma el fenol como el compuesto de referencia, se tiene que para los otros dos compuestos, se manifiesta la influencia del grupo nitro y la influencia del cloro como sustituyentes del fenol, los cuales afectan el proceso de adsorción sobre los materiales carbonosos. Una vez se han determinado las entalpías de inmersión, y dado que se han mantenido las mismas condiciones experimentales, se puede calcular la contribución entálpica, que tienen los sustituyentes, en la adsorción para cada uno de los carbones activados estudiados; dicha contribución se obtiene como la diferencia entre la entalpía de inmersión en solución acuosa de fenol menos la que se obtiene en solución acuosa del 3cloro fenol y del 4-nitro fenol. La Figura 7 muestra la contribución entálpica de la adsorción del 3-cloro fenol y el 4-nitro fenol en los carbones activados en relación con el $\mathrm{pH}_{\mathrm{PzC}}$ dado que este caracteriza la carga de los sólidos y muestra su influencia en el proceso de adsorción.

Como la entalpía de inmersión de los carbones activados en disoluciones acuosas de 3-cloro fenol son menores que las del fenol, se obtiene que la contribución entálpica del sustituyente - $\mathrm{Cl}$, cuando está presente en el anillo fenólico, en el proceso de adsorción es negativa y aumenta cuando aumenta el valor del $\mathrm{pH}$ Pzc. Para el caso del grupo $-\mathrm{NO}_{2}$ como sustituyente del anillo, se presenta que la contribución entálpica es positiva y de igual forma aumenta al aumentar el valor del $\mathrm{pH}_{\mathrm{Pzc}}$. El comportamiento que tiene la relación es lineal, aunque se observa que los puntos están en algunos casos distantes de la tendencia; los coeficientes de correlación obtenidos son de 0.6127 para el caso del 3-cloro fenol y de 0.6202 para el caso del 4-nitro fenol. El cálculo de la contribución entálpica de cada compuesto en los diferentes carbones activados permite observar, para el caso del 4-nitro fenol, que ésta es mayor para el carbón activado CACu, preparado a partir de cuesco de palma africana, y que no ha mostrado ser el adsorbente con mayor adsorción para este compuesto.

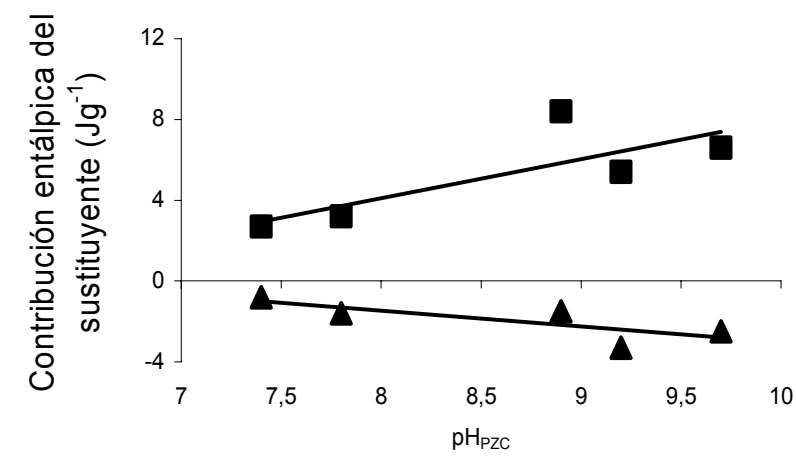

Fig. 7: Contribución entálpica del sustituyente en función del $\mathrm{pH}_{\mathrm{PZC}}$. 4-nitro fenol, $\mathbf{\Delta}$ 3-cloro fenol.

\section{CONCLUSIONES}

Se estudia las interacciones entre cinco muestras de carbón activado, obtenidas a partir de diferentes materiales lignocelulósicos y con grado de activación moderado, 20 por ciento, y disoluciones acuosas de fenol y 3-cloro fenol mediante la determinación de las entalpías de inmersión.

Se establece que los carbones activados obtenidos son de carácter básico y presentan valores para el $\mathrm{pH}$ en el punto de carga cero, $\mathrm{pH}_{\mathrm{PzC}}$, que varían entre 7.4 y 9.7 y contenidos de basicidad total mayores en todos los casos que los valores obtenidos para la acidez total. Igualmente se determina 
la entalpía de inmersión de los carbones activados en disoluciones $0.1 \mathrm{M}$ de $\mathrm{NaOH}$ y $\mathrm{HCl}$ con valores mayores para la entalpía de inmersión en $\mathrm{HCl}$ que se encuentran entre 32.6 y $68.3 \mathrm{Jg}^{-1}$.

Las entalpías de inmersión de los carbones activados en disolución de fenol se hallan entre 7.60 y $13.9 \mathrm{Jg}^{-1}$ y para el caso del 3-cloro fenol están entre 6.80 y $11.4 \mathrm{Jg}^{-1}$. Los resultados obtenidos se comparan con entalpías de inmersión y cantidad adsorbida para el 4-nitro fenol, resultados que se han obtenido con anterioridad; y que presenta valores mayores de entalpías de inmersión para todas las muestras.

Se observa que la cantidad de los compuestos fenólicos adsorbidos disminuye con el contenido de grupos ácidos y que la entalpía de inmersión de los carbones activados en soluciones acuosas de los compuestos mencionados aumenta cuando se incrementa el valor del pH en el punto de carga cero, $\mathrm{pH}_{\mathrm{PzC}}$.

\section{AGRADECIMIENTOS}

Los autores agradecen al convenio marco existente entre la Universidad Nacional de Colombia y la Universidad de Los Andes. Un agradecimiento especial, al Fondo de investigaciones de la Facultad de Ciencias de la Universidad de Los Andes y su proyecto semilla.

\section{REFERENCIAS}

Amaya, B.A. y Rancel, D.O., Obtención de carbón activado a partir de residuos lignocelulósicos y diseño de un adsorbedor para la remoción de $\mathrm{Cr}^{6+}$ y $\mathrm{Pb}^{2+}$ en corrientes acuosas. Trabajo de grado. Facultad de Ingenieria. Universidad de los Andes, Bogotá, Colombia (2004)

Boehm, H.P., Advances in Catalysis. Eley, D.D.H. Pines., P.B. Weisz, 2 Edición, pp 192., Academic Press, New York. (1966)

Briceño, N., Caracterización de carbones activos obtenidos de cuesco de palma africana por activación cin dióxido de carbono, Trabajo de grado. Departamento de Química, Universidad Nacional de Colombia. (2002)

Cartula, A., Martín, M., Molina, M., R. Rodríguez, F. y Torregrosa. R., Adsorption of Substituted Phenols on Activated Carbon. J. Colloid and Interface Science 24, 528-534 (1998)

De Zuane, J.P.E. Handbook of drinking water quality. 2th ed., Wiley., New Cork. 1997

Diaz, C.M., Briceño, N., Baquero, M.C., Giraldo, L. y Moreno J.C., Caracterización textural y química de carbón activado obtenido a partir de cuesco de palma africana a diferentes condiciones de temperatura y tiempo de carbonización. Revista Colombiana de Química, 31(2), 119-129 (2002)

Diaz, C.M., N. Briceño., Baquero, M.C., Giraldo, L. y Moreno, J.C., Influence of temperature in the proceses of carbonization and activation with $\mathrm{CO}_{2}$ in the obtainment of activated carbon from african palm pit. Study of the modification of characterization parameters. Internet Journal of Chemistry, 6, GMT 13:52:00 (2003)

Giraldo, L., Moreno, J.C. y Huertas, J.I., Heats Conduction Micro-Calorimeter With Metallic Reaction Cells. Instrumentation Science \& Technology. 30 (2), 177-186 (2002)

Giraldo, L. y Moreno, J.C., Determinación de la entalpía de inmersión de carbón activado en soluciones acuosas de fenol y su relación con la capacidad de adsorción. Revista Colombiana de Química, 32(1), 45-54 (2003)

Giraldo, L. y Moreno, J.C., Determinación de la entalpía de inmersión y capacidad de adsorción de un carbón activado en soluciones acuosas de plomo. Rev. Col. Quim. 33(2), 87-97 (2004) 
Lopez, R. M., Stoeckli, F., Moreno, C.C. y Carrasco, M.F., On the Characterization of Acidic and Basic Surface Sites on Carbons by Various Techniques. Carbon, 37, 1215-1221 (1999)

McGuire, M.J. y Suffet, I.H., Treatment of water by granular activated carbon. $2^{\text {a }}$ Edición, 29-33, American Chemical Society, Washington D.C. (1983)

Montes-Morán, M.A., Suárez, D., Menéndez, J.A. y Fuente, E., On the nature of basic sites on carbon surfaces: an overview. Carbon 42, 1219-1225 (2004)

Moreno-Castilla, C., Rivera-Utrilla, J., López-Ramón, M.V., Carrasco-Marín, F., Adsorption of some substituted phenols on activated carbons from bituminous coal, Carbon, 33 845-851 (1995)

Moreno-Castilla, C., Adsorption of organic molecules from aqueous solutions on carbon materials. Carbon. 42, 83-94 (2004)

Mozia, S., Tomaszewska, M. y Morawski, W., Studies on the effect of humic acids and phenols on adsorption, Water Research, 39(2), 501 (2005)

Navarrete, L.F., Giraldo, L., Moreno, J.C. y García, V., The influence of superficial chemistry in the immersion enthalpy of activated carbon in aqueous solutions of phenol and 4-nitro phenol. $2^{\text {nd }}$. International symposium on calorimetry and chemical thermodynamics. Sao Pedro, Brazil. Abril (2006)

Nemerow, N.L., Dasgupta, A., Industrial and hazarduos waste treatment, Van Nostrand Reinhold, New York. (1991)

Nevskaia, D.M., Castillejos-López, E., Guerrero-Ruiz, A. y Muñoz A. Effects of the surface chemistry of carbons materials on the adsorption of phenol-aniline mixtures from water. Carbon, 42(3), 653 (2004)

Otero, M., Zabkova M. y Rodríguez A., Adsorptive purification of phenol wastewater. Chem. Eng. Journal, 110(1), 101 (2005)

Radovic, L.R., C.C. Moreno. y U.J. Rivera., Carbon Materials as Adsorbents in Aqueous Solutions. In Chemistry and Physics of Carbon. A Serie of Advances. 1ª Editión, 293-297, Marcel Dekker, New York (2000)

Rodríguez, R.F., Activated carbon: structure,characterization, preparation and applications. En: Introduction to carbon technologies. Pp 60 Universidad de Alicante (Publicaciones). Alicante, España. (1997)

Roostaei, N. y Tezel, H., Renoval of phenol from aqueous solutions by adsorption. J. Environm. Manag., 70(2), 157 (2004)

Toles, C.A., W.E. Marshall y M.M. Johns., Surface functional groups on acid-activated nutshell carbons. Carbon, 37, 1207-1214 (1999)

Tseng, R.L., F.C. Wu y R.S. Juang., Liquid-fase adsorption of dyes and phenols using pinewoodbased activated carbons. Carbon 41, 487-495 (2003) 